\title{
Los anhelos a futuro de estudiantes de maestría en espacios universitarios asimétricos de la Ciudad de México y Guadalajara
}

\section{The future aspirations of Masters' degree students in asymmetrical university spaces in Mexico City and Guadalajara}

DOI: https://doi.org/10.32870/dse.v0i24.1057

\section{Karla Teresa Camacho Rodríguez*}

\begin{abstract}
Resumen
Este artículo analiza las aspiraciones de futuro de mujeres y hombres estudiantes de la maestría de derecho, administración e ingeniería de una muestra no representativa de Espacios Universitarios Consolidados y en Vías de Consolidación de la Ciudad de México y Guadalajara. El análisis muestra que entre las y los maestrantes los anhelos económicos (ganar mucho dinero y alcanzar estabilidad económica) y los académicos (estudiar un doctorado, ser profesor/a y desarrollar investigación) han perdido importancia; mientras que sus principales aspiraciones de futuro se configuran en torno a los anhelos social-relacionales y laborales, vistos a través de la intensión de alcanzar estabilidad económica y reconocimiento como profesionistas. En relación con el sexo, mientras que entre las mujeres se anhelan experiencias, abreviadas en las oportunidades para viajar, para los hombres tener prestigio es el principal anhelo.
\end{abstract}

Palabras clave: aspiraciones de futuro - desigualdades - espacios universitarios asimétricos - anhelos estudiantes de maestría.

\begin{abstract}
This article analyzes the aspirations for the future of students of the Masters' degree programs of Law, Business Management, and Engineering from a non-representative sample of Consolidated University Spaces and Spaces in a Process of Consolidation in Mexico City and Guadalajara. Our analysis shows that economic (earning a lot of money and achieving economic stability) and academic aspirations (studying a PhD, teaching and doing research) have lost importance, while the students' main aspirations for the future revolve around the social-relational and the labor market, as seen in their aim of achieving financial stability and recognition as professionals. While female students seek to have new experiences and opportunities to travel, men seek prestige as a main aim.
\end{abstract}

Keywords: Aspirations for the future - inequality - asymmetric university spaces - yearning - master degree students.

* Doctora en Ciencias Antropológicas y especialista en temas de desigualdad, juventudes y clases sociales. Escuela Nacional de Antropología e Historia. México. karlacamacho26@gmail.com 


\section{Introducción}

Las aspiraciones de futuro es un concepto multidimensional que se refiere a las afirmaciones de voluntad, a definiciones de lo deseable y a un marco de orientación conductual que evidencia aquello que están dispuestos a hacer los sujetos para que esto ocurra (Appadurai, 2004). Entre sus características principales están el ser útil a la hora de considerar el género, el nivel socioeconómico y la raza como variables que pueden incidir en la forma en que se percibe el futuro, y en la búsqueda de entender cómo es que ciertos rasgos de la personalidad inciden en el tipo de orientación hacia el futuro (Omar, 2005; Delgado et al., 2010).

Su uso en los estudios sobre juventudes ha permitido, entre otras cosas, conocer las actitudes y expectativas con las que se enfrentan estas poblaciones (Omar, 2005; Delgado et al., 2010). Cuando las perspectivas de futuro se han examinado en el contexto escolar, ha sido visible la importancia que tiene para el estudiantado la obtención de grados, alcanzar títulos, prepararse para la vida laboral y, de manera general, conocer las principales pautas con las que conciben el futuro en el cruce con su experiencia escolar (Gaeta, Reyes, Gonzales et al., 2020; Barrera, Sotelo, García, 2018; Corica, 2012, entre otros).

En algunas investigaciones se ha analizado la influencia que tiene el tipo de establecimiento educacional al que concurren los jóvenes en la definición de sus aspiraciones, de las posibilidades que tienen de alcanzar sus objetivos, de los factores que pueden incidir en el logro o fracaso de sus planes, y de cómo se articulan la educación y el trabajo en los planes de futuro (Sepúlveda, Baldevenito, 2014). Así, y considerando las múltiples variables que tienen influencia en la construcción de las aspiraciones, se ha dado cuenta no sólo de los factores externos que inciden en la delimitación de los anhelos de futuro en las y los jóvenes, sino también de las emociones que experimentan en la etapa de construcción de éstas.

El análisis de las aspiraciones, vistas también como representaciones construidas por los individuos y los grupos acerca de lo que se desea para el futuro, ha mostrado que es importante distinguir entre las expectativas que refieren a lo que se cree que ocurrirá en el futuro dadas las tendencias actuales, y las aspiraciones que apuntan hacia lo que se desea que ocurra y se está dispuesto a hacer para ello (PNUD, 2000: 58).

No obstante, aunque desde hace años se ha observado un aumento constante en la matrícula y los programas de formación de posgrado, y que el hecho de acceder a un nuevo nivel educativo puede implicar la construcción de nuevas aspiraciones o bien la modificación o consolidación de otras, parece que el interés por comprender las aspiraciones de futuro del estudiantado se rompe de manera súbita con los estudiantes de licenciatura.

Entendiendo que las y los estudiantes de posgrado poseen mayores capitales, educativos y relacionales, que han acumulado experiencias en el espacio universitario y laboral y que tienen parámetros diferenciados que les permiten ampliar el espectro de sus decisiones, y en esencia, una mayor agencia que les permite asentar en distintos planos de su vida sus aspiraciones a 
futuro (García-Castro, Bartolucci, 2007), en este artículo se analizan las aspiraciones de futuro de mujeres y hombres estudiantes de maestría.

Para ello, comenzaremos exponiendo un marco de análisis donde se presenta el concepto de aspiración y la propuesta de Villa, Canales y Hamui (2020) sobre la asimetría de los Espacios Universitarios (EU). Posteriormente, se analizan algunos resultados de una encuesta aplicada entre estudiantes de las carreras de derecho, administración e ingeniería de Espacios Universitarios Consolidados y en Vías de Consolidación de la Ciudad de México y Guadalajara, para dar cuenta de las diferencias en los anhelos de futuro de acuerdo con la carrera, el sexo y el EU de un grupo de estudiantes de maestría. Finalmente, se presentan algunas conclusiones y propuestas para profundizar en los hallazgos.

\section{Marco de análisis}

\section{Las aspiraciones}

En la década de los noventa la idea de elevar las aspiraciones comenzó a percibirse como un punto central en los proyectos de justicia social de países como Australia y Reino Unido (Sellar, Gale, Parker, 2011). Si bien el "aumento de las aspiraciones" como tendencia política ha sido criticado por su sesgo neoliberal, en la medida en que al articularse con la idea de elevar el capital social se busca que los ciudadanos, y no el Estado, sean quienes maximicen sus oportunidades asumiendo la responsabilidad de su progreso socioeconómico a través de la inversión en bienes como la educación, se ha abierto un debate sustancial en torno a las concepciones de la aspiración.

Desde la perspectiva de Appadurai (2004), las aspiraciones son afirmaciones de voluntad, definiciones de lo deseable y, por ello, un marco de orientación conductual que revela lo que se está dispuesto a hacer para que eso ocurra. Así, aunque la aspiración ha sido mayormente trabajada desde la ciencia económica, este concepto debe ser abordado como propiamente cultural, en la medida en que en él se incluyen no sólo mundos imaginados por grupos sociales, sino también normas de acción y un sentido común que orienta el quehacer de los sujetos.

La aspiración implica la narración y navegación de las relaciones entre los deseos, las preferencias inmediatas y los contextos culturales más amplios. Appadurai (2004) sugiere incluso que puede entenderse mejor como una capacidad, es decir, como una aptitud que se puede desarrollar más cuando se tiene una relación poderosa con el propio futuro, y el futuro colectivo más amplio. Cuando los individuos participan en grupos cuya posición le otorga una mayor influencia, puede incluso desarrollar mayormente esta capacidad. En las propias palabras de Appadurai:

La aspiración es la capacidad desigualmente distribuida de los diferentes colectivos sociales para articular poderosamente sus visiones de la buena vida; para expresar, y hacer que otros reconozcan los va- 
lores, creencias y normas que dan textura a estas visiones; para acceder y utilizar los recursos necesarios para realizarlas. La capacidad de aspirar implica tanto la capacidad de realizar aspiraciones concretas, como el poder colectivo de conformar contextos culturales en los que se forman diferentes deseos y se valoran como aspirativos (Appadurai, 2004: 69).

Pero no sólo la capacidad de aspirar o de realizar los futuros imaginados, sino también la delimitación de lo que se reconoce como aspiración se articula con la lógica de la desigualdad (Sellar, Gale, Parker, 2011). ${ }^{1}$ Por ello es importante pensar de forma más extensa y multidimensional en los factores que conforman y estimulan los futuros imaginados por las personas.

En la intersección de los contextos (familiares, sociales o de grupo) y otras influencias mediadas por lo social, como los medios de comunicación o las redes, puede entenderse cómo se forman las identidades, comunidades y los proyectos de vida. Desde su trabajo en el ámbito educativo, Sepúlveda y Baldevenito (2014) indican que para el análisis de las aspiraciones resulta fundamental estudiar la subjetividad de los sujetos (sus proyectos personales, ambiciones o deseos) y su rol activo en la definición de itinerarios y trayectorias distintivas, equilibrando una mirada que hasta ahora ha estado mayormente centrada en la dimensión institucional del sistema educativo. Porque las aspiraciones también pueden concebirse como anhelos o sueños, en la medida en que son las representaciones que se hacen los individuos y los grupos acerca del estado de cosas, personales o sociales, que desean para el futuro y que caracterizan como "lo mejor".

Sobre los factores que forjan las aspiraciones se ha discutido mucho. No obstante, de acuerdo con Figueroa, Padilla y Guzmán (2015), las variables más significativas en la construcción de las aspiraciones educativas entre los jóvenes son: el sexo, la edad, el desempeño académico y otras variables relacionadas con la familia (como la escolaridad y la ocupación de los padres, la interacción entre padres e hijos y las expectativas y aspiraciones de los padres hacia la educación superior).

Para Jiménez (2004), las variables que intervienen en la formación de los itinerarios asociados a las aspiraciones son, además: a) las condiciones sociales: contexto social o territorio económico, clase social, origen familiar y sexo; $b$ ) la oferta pública y privada de formación y empleo, y mecanismos y políticas de inserción; y c) las aptitudes, actitudes y estrategias del sujeto: microclima familiar, grupo de iguales, etc., en cuanto constructores de autoimagen, sistema de valores, actitudes y expectativas.

En síntesis, podemos entender las aspiraciones como capacidades ligadas a un marco conductual de construcción compleja en el que intervienen variables individuales (aptitudes, ac-

1 Para explicar la importancia de la construcción de las aspiraciones válidas, Sellar, Gale y Parker (2011) recuperan el ejemplo de la Educación Superior al observar cómo ésta conlleva en la sociedad actual un imaginario sobre la conveniencia inherente de los estudios universitarios. La aspiración, sostienen, es sinónimo de búsqueda de la Educación Superior, quien no se adhiere a este imaginario corre el riesgo de ser considerado de bajas aspiraciones. 
titudes, estrategias), sociales (condiciones sociales) y políticas e institucionales; como un conjunto de anhelos y deseos que orientan las acciones de los sujetos y que se van construyendo a través de itinerarios, y como configuraciones de deseos y futuros de aquello que los individuos consideran como "lo mejor"

\section{Espacios universitarios consolidados y espacios universitarios en vías de consolidación}

Villa, Canales y Hamui (2017) sostiene que los espacios universitarios en México son asimétricos, porque ofrecen condiciones distintas a sus estudiantes, de acuerdo con las características del alumnado, la planta académica, sus programas de licenciatura y posgrado, de su infraestructura, su historia y del propio prestigio que se han creado. Las diferencias entre estos espacios universitarios son relevantes porque responden a una configuración de educación superior compuesta por circuitos diferenciados de calidad, que propician a su vez que funcionen como estructuras de desigualdades. ${ }^{2}$

Las diferencias institucionales en los recursos académicos, que redundan en condiciones, calidad y estructuras de oportunidades diversas, dividen los Espacios Universitarios en Consolidados y en Vías de Consolidación. Los Espacios Universitarios Consolidados (EUC), que pueden ser tanto públicos como privados, se caracterizan por darle un papel preponderante a la investigación y al intercambio nacional e internacional de conocimientos científicos y tecnológicos, tanto de profesores como de estudiantes. Se caracterizan también por ofrecer estudios de posgrado, publicar los resultados de sus investigaciones en editoriales de prestigio y por pertenecer y formar redes en todas las áreas de conocimiento.

Los Espacios Universitarios denominados en Vías de Desarrollo (EUV), que igualmente pueden ser públicos o privados, priorizan la atención de la demanda local de Educación Superior y están centrados en la licenciatura. Este grupo se caracteriza por la concentración de su oferta educativa en áreas de conocimiento rentables, y por la disparidad en sus recursos financieros, institucionales y académicos, en muchos casos signados por la baja calidad de los servicios que ofrecen.

Estas tendencias, afirman Villa, Canales y Hamui (2017), además de asimetrías entre los espacios universitarios, propician una segmentación social entre el estudiantado; cuestión que, aunada al género, la carrera, la etnia y la clase social de origen pueden ampliar o reducir el horizonte de la capacidad de aspirar de las y los estudiantes.

2 Autores como Kessleer (2003) y Tiramonti (2004) han mostrado también que la desigualdad existe en la oferta académica, que se refleja en la infraestructura, en los recursos de las escuelas, en el tipo de educación que reciben los alumnos, en las características de las instituciones o bien en la formación docente. 
Los valores de las instituciones, carreras y género. Otros principios de las desigualdades en la Educación Superior

Como otra dimensión de la desigualdad en la ES, podemos observar que las instituciones de educación superior ostentan filosofías y valores diferenciados. ${ }^{3}$ Los programas de estudio y la caracterización de los perfiles de los estudiantes que se forman en las IES conllevan concepciones diferenciadas de lo que significa ser universitario y, de manera general, ser profesionista en las diversas áreas de estudio. Convertirse en "miembro" de la institución universitaria requiere aprender el oficio de estudiante, lo que a su vez significa adquirir disposiciones y actitudes hacia el conocimiento, aprender las reglas de la vida universitaria, además de competencias para desenvolverse en la actividad intelectual y profesional enmarcada en la visión de las instituciones (Guillen, 2009).

Trabajos como el de Luna, Valle y Osuna (2008), el de Hirsch y Pérez (2005) o bien el de Oakley y Cocking (2001), revelan que los roles profesionales están construidos por sistemas de valores diferenciados que sirven a los objetivos de las profesiones. La jerarquización de la importancia que le dan las y los estudiantes a los valores que se orientan a la contribución con el ejercicio profesional en el mejoramiento de la calidad de vida de la sociedad o aquellos demandados en la sociedad del conocimiento como trabajar en equipo, no son igualmente relevantes para el estudiantado, porque depende del área de conocimiento en la que se forma (Luna, Valle, Osuna, 2008). Oakley y Cocking (2001) afirman inclusive que las IES necesitan urgentemente trabajar en la formación de valores ético-profesionales, en la medida en que estos suelen ser poco significativos entre el estudiantado. ${ }^{4}$

Por otro lado, el papel del género en las desigualdades en el contexto universitario también se ha reflexionado mucho. Si entendemos el género "como una identidad social, que confiere una jerarquía de valores; un concepto de lo que es el trabajo; una manera de ser, una manera de responder a los estímulos; una forma de actuar y de aspirar a determinada cosa y nada más" (Hierro, 1996: 41), hallamos que las relaciones de género en la educación, y los factores que intensifican las desigualdades entre hombres y mujeres, resultan determinantes en la construcción de las aspiraciones del estudiantado.

Así, por ejemplo, aunque en los últimos años se ha observado un aumento progresivo en la matrícula de las mujeres a nivel posgrado, los motivos de elección de las carreras y los propios perfiles de los programas de estudio continúan estando sujetos a desigualdades de género. ${ }^{5}$

$3 \mathrm{Si}$ bien la literatura sobre los valores es también bastante amplia, hay que entenderlos como guías del comportamiento y que orientan las acciones humanas en las situaciones concretas de la vida (Oser, 1997 en Luna, Valle, Osuna, 2008).

4 López (2017) apoya esta idea al afirmar que la idea del "buen profesional" que se va construyendo en el paso por la universidad, delinea el comportamiento profesional; por lo que la construcción de proyectos educativos vinculados a las necesidades sociales y la formación de perfiles profesionales competentes, socialmente comprometidos y con vocación hacia la justicia, es decir, profesionistas éticos, debiera ser un objetivo primordial para las IES.

5 Diversos estudios sobre elecciones académicas y género muestran cómo las decisiones académicas y profesionales están sesgadas por el sexo. En líneas generales, se menciona que las mujeres se inclinan por las familias profesionales de administración, sanidad, imagen personal, etc., mientras que los hombres por las familias profesionales más técnicas. 
Ángeles (2016) ha destacado incluso que hay una relación entre la temporalidad individual de la población que estudia maestría con los roles de género, en la medida en que mujeres que egresan de carreras universitarias de áreas como ciencias sociales, administración, derecho y educación suelen ingresar a maestrías de la misma área, mientras que los hombres enfrentan una disyuntiva entre transitar a una maestría de la misma área o laborar. Ello ha creado una diferencia entre los hombres y las mujeres en cuanto a la edad de ingreso a este nivel de posgrado.

Por ello, vale destacar que las condiciones materiales y objetivas que diferencian y jerarquizan a las IES, soportan además condiciones simbólicas, valorativas y prácticas, como el género y los valores diferenciados de las carreras e instituciones, que potencializan las desigualdades estructurales en las que participan y construyen sus aspiraciones las y los estudiantes.

\section{Metodología}

Los datos que se analizan en este artículo provienen del trabajo empírico que se realizó como parte de la primera fase del proyecto "Las aspiraciones de futuro de los estudiantes de Educación Superior desde una perspectiva de desigualdades multidimensionales". En esta fase se aplicaron un total de 3,659 encuestas a estudiantes de seis espacios universitarios, tres públicos y tres privados, de la Ciudad de México y Guadalajara. Estos espacios, como se muestra a continuación, fueron clasificados en Espacios Académicos Consolidados (EAC) y espacios Académicos en Vías de Consolidación (EAV). ${ }^{6}$

6 Los EU de este estudio fueron seleccionados con base en tres rasgos que permitieron tipificarlos y analizarlos a través de sus asimetrías, además de observar si la diversidad de sus características intervienen en las aspiraciones de las y los estudiantes. El primero fue la ubicación socioespacial y las bases fundacionales. Los EU seleccionados están ubicados en Ciudad de México y Guadalajara, que los sitúan en un entramado específico de relaciones que, junto con el tipo de fundación vinculada con una ideología y con requerimientos de los grupos sociales a los que desean responder, configuran tipos institucionales con normas y valores específicos. El segundo rasgo es el tipo de financiamiento que sostiene a los EU, razón por la que pueden pertenecer a dos subcampos con características y dinámicas distintas: el subcampo público, que congrega a universidades laicas, gratuitas y autónomas, cuyo financiamiento proviene del Estado (ya sea federal o estatal), que albergan un número considerable de estudiantes, programas y académicos, y que es donde se encuentra la mayoría de los estudios de doctorado y una parte importante de la investigación científica y tecnológica que se realiza en el país. Este subcampo congrega un conjunto institucional estratificado y segmentado tanto vertical como horizontalmente (Villa, Canales, Hamui, 2017: 163). El subcampo privado alberga además una diversidad de IES que se diferencian por su tamaño, por la calidad de los servicios que ofrecen, por su orientación y por el tipo de grupo social al que se dirigen; tradicionalmente han recibido su financiamiento de particulares (sean corporaciones religiosas, grupos de empresarios $u$ otros) y por el cobro de colegiaturas. La mayoría de sus establecimientos son pequeños y se les puede denominar "incompletos" o"parciales", "pues tienen una reducida oferta de carreras y no tienen infraestructura de investigación". A ellas ingresa una población principalmente de los estratos medios y bajos, que además en muchos casos no pudieron ingresar a la universidad pública (Muñoz, 2009: 262-263; Acosta, 2005: 8). Existen asimismo las universidades privadas de alto desarrollo académico, a las que acceden las élites; es decir, la educación superior privada también está estatificada y conformada por espacios universitarios diversos, jerarquizados y asimétricos." (Villa Lever, Canales, Hamui, 2017: 171-172). El último rasgo fue el grado de desarrollo académico que clasifica a los EU en consolidado y en vías de consolidación, y cuyas caracteristicas principales describimos anteriormente. 


\begin{tabular}{|l|l|}
\hline $\begin{array}{l}\text { Nombre de Institución de Educa- } \\
\text { ción Superior (IES) }\end{array}$ & Tipo de espacio \\
\hline $\begin{array}{l}\text { Universidad Nacional Autónoma de } \\
\text { México (UNAM) }\end{array}$ & $\begin{array}{l}\text { Espacio Universitario Público Con- } \\
\text { solidado; Ciudad de México (EUPUC- } \\
\text { CDMX) }\end{array}$ \\
\hline $\begin{array}{l}\text { Universidad Autónoma de la Ciudad de } \\
\text { México (UACM) }\end{array}$ & $\begin{array}{l}\text { Espacio Universitario Público en } \\
\text { Vías de Consolidación; Ciudad de } \\
\text { México (EUPuV-CDMX) }\end{array}$ \\
\hline Universidad Iberoamericana (IBERO) & $\begin{array}{l}\text { Espacio Universitario Privado Con- } \\
\text { solidado; Ciudad de México (EUPriC- } \\
\text { CDMX) }\end{array}$ \\
\hline Universidad del Valle de México (UVM) & $\begin{array}{l}\text { Espacio Universitario Privado en } \\
\text { Vías de Consolidación; Ciudad de } \\
\text { México (EUPriV-CDMX) }\end{array}$ \\
\hline Universidad de Guadalajara (UDG) & $\begin{array}{l}\text { Espacio Universitario Público Con- } \\
\text { solidado; Guadalajara (EUPuC-GDL) }\end{array}$ \\
\hline Universidad del Valle de Atemajac \\
(UNIVA) & $\begin{array}{l}\text { Espacio Universitario Privado en } \\
\text { Vías de Consolidación; Guadalajara } \\
\text { (EUPriV-GDL) }\end{array}$ \\
\hline
\end{tabular}

El cuestionario que se aplicó a las y los estudiantes se constituyó de preguntas cerradas y de opción múltiple. En él se abordaron cuatro variables: características sociodemográficas, antecedentes académicos, aspiraciones laborales, educativas y de ciudadanía y experiencia laboral. En esta encuesta participaron además estudiantes que se encontraban en tres momentos: primero y último año de licenciatura y durante la maestría. La información se reunió a partir de visitas a distintas sedes de las universidades seleccionadas durante el primer semestre del año 2019-2020 y para el análisis de datos se utilizó el programa SPSS. El cuestionario constó de 68 preguntas, aunque en este análisis nos centraremos principalmente en la pregunta de opción múltiple: ¿¿Cuáles son sus principales anhelos?” y a parir de ella retomaremos otras respuestas

7 La misión que las páginas de las universidades consideradas en este estudio destacan como parte de sus proyectos educativos es: 1) UNAM: Tiene como propósito primordial estar al servicio del país y de la humanidad, formar profesionistas útiles a la sociedad, organizar y realizar investigaciones, principalmente acerca de las condiciones y problemas nacionales, y extender con la mayor amplitud posible, los beneficios de la cultura; 2) UACM: Se propone desarrollar un proyecto innovador, constituir una comunidad académica, y garantizar la libertad y la pluralidad de pensamiento; 3) IBERO: Su objetivo es ayudar al desarrollo social, local, regional y nacional, y formar una sociedad más justa, humana y fraterna, a través de la docencia, de los programas de formación profesional, vinculación, incidencia social e investigación con los que cuenta esta casa de estudios; 4) UVM: Se plantea dar acceso a una educación de calidad en un ambiente crítico, diverso e integrado a una red global de universidades, formar profesionales competentes e íntegros y aportar las capacidades institucionales al desarrollo de la región y el país; 5) UDG: Busca promover la investigación científica y tecnológica, así como la vinculación y extensión para incidir en el desarrollo sustentable e incluyente de la sociedad. Es respetuosa de la diversidad cultural, honra los principios humanistas, la equidad, la justicia social, la convivencia democrática y la prosperidad colectiva; 6) UNIVA: Su misión es formar personas vinculadas responsablemente al ser y a los anhelos de la comunidad que desde una perspectiva cristiana y católica colaboren al desarrollo sustentable de la nación, a través de un desempeño profesional de alta calidad, congruente con los avances científicos y tecnológicos contemporáneos. 
del cuestionario que nos permitan comprender las aspiraciones de futuro de las y los estudiantes de maestría.

En el grupo de estudiantes de maestría, en el cual nos centraremos, se incluye un total de 853 mujeres y hombres cuya distribución, según el Espacio Universitario, la carrera que cursan y el género, puede observarse en la tabla que se presenta a continuación: ${ }^{8}$

\begin{tabular}{|c|c|c|c|c|}
\hline \multicolumn{5}{|c|}{$\begin{array}{l}\text { Número de estudiantes de maestría por universidad, sexo y } \\
\text { carrera }\end{array}$} \\
\hline & Carrera & & exo & Total \\
\hline Universidad & & Mujer & Hombre & \\
\hline EUPUC-CDMX & \multirow{6}{*}{ Administración } & 126 & 104 & 230 \\
\hline EUPUV-CDMX & & 6 & 6 & 12 \\
\hline EUPUC-GDL & & 31 & 84 & 115 \\
\hline EUPriC-CDMX & & 38 & 52 & 90 \\
\hline EUPriV-CDMX & & 32 & 25 & 57 \\
\hline EUPriV-GDL & & 1 & 7 & 8 \\
\hline Subtotal & & 234 & 278 & 512 \\
\hline $\begin{array}{l}\text { EUPUC-CDMX- } \\
\text { UNAM }\end{array}$ & \multirow{6}{*}{ Derecho } & 24 & 29 & 53 \\
\hline $\begin{array}{l}\text { EUPUV-CDMX- } \\
\text { UACM }\end{array}$ & & 19 & 12 & 31 \\
\hline $\begin{array}{l}\text { EUPUC-GDL- } \\
\text { UDG }\end{array}$ & & 27 & 38 & 65 \\
\hline $\begin{array}{l}\text { EUPriC-CDMX- } \\
\text { IBERO }\end{array}$ & & 28 & 7 & 35 \\
\hline $\begin{array}{l}\text { EUPriV-CDMX- } \\
\text { UVM }\end{array}$ & & 9 & 13 & 22 \\
\hline EUPriV-GDL & & 2 & 4 & 6 \\
\hline Subtotal & & 109 & 103 & 212 \\
\hline
\end{tabular}

8 Como puede observarse, en esta fase participaron un total de 303 estudiantes del EUPuC-CDMX, 71 del EUPuV-CDMX, 207 del EUPuC-GDL, 147 del EUPriC-CDMX, 101 del EUPriv-CDMX y 19 del EUPriv-GDL. La diferencia entre el número de estudiantes por institución proviene de que, en un primer momento, se acudió a las instituciones para conocer cuál era el número de estudiantes por programa y nivel de estudios, pero los datos obtenidos fueron muy heterogéneos. Ello impidió tener un marco muestral confiable y por ello se decidió trabajar con una muestra no probabilística, que si bien no tiene representatividad, sí nos ayuda a describir distintas realidades y plantear hipótesis. Los espacios universitarios incluidos en esta investigación fueron además seleccionados como casos genéricos, en la medida en las que tienen una representatividad analítica que permite comprobar distintas proposiciones y son relevantes porque muestran no sólo la situación de cada espacio, sino también la de otras universidades con las que comparten distintas características. 


\begin{tabular}{|c|c|c|c|c|}
\hline $\begin{array}{l}\text { EUPUC-CDMX- } \\
\text { UNAM }\end{array}$ & \multirow{6}{*}{ Ingeniería } & 2 & 20 & 22 \\
\hline $\begin{array}{l}\text { EUPUV-CDMX- } \\
\text { UACM }\end{array}$ & & 8 & 21 & 29 \\
\hline $\begin{array}{l}\text { EUPUC-GDL- } \\
\text { UDG }\end{array}$ & & 5 & 24 & 29 \\
\hline $\begin{array}{l}\text { EUPriC-CDMX- } \\
\text { IBERO }\end{array}$ & & 8 & 14 & 22 \\
\hline $\begin{array}{l}\text { EUPriV-CDMX- } \\
\text { UVM }\end{array}$ & & 7 & 15 & 22 \\
\hline EUPriV-GDL & & 1 & 4 & 5 \\
\hline Subtotal & & 31 & 98 & 129 \\
\hline Total & & 374 & 479 & 853 \\
\hline
\end{tabular}

Algunas de las características generales de este grupo son que la moda de edad de las y los maestrantes al momento de la aplicación de la encuesta fue de 27 años, mientras que el promedio de edad fue de 31 años. El rango de edad, no obstante, osciló entre los 20 y 65 años, aunque se concentró el mayor número de estudiantes (45\%) en el rango de los 30 y 39 años.

En relación con sus condiciones socioeconómicas, del total de estudiantes de esta muestra, $1.3 \%$ considera que su familia pertenece a una clase social alta, $36 \%$ se asume como de clase media alta, 56.3\% considera que pertenece a una clase social media baja y 5.4\% indica que pertenece a una clase social baja. ${ }^{9}$ Los maestrantes que indican que pertenecen a una clase alta son en su mayoría hombres de la carrera de administración de instituciones como el EUPriC-CDMX, el EUPUC-CDMX y el EUPriV-CDMX; es decir, tres Espacios Universitarios de la Ciudad de México, dos privados (uno consolidado y otro en vías de consolidación) y el Espacio Público Consolidado de esta ciudad. En las posiciones de clase media alta y baja podemos encontrar estudiantes de las distintas instituciones y cierta paridad en cuanto al género.

En relación con el ingreso, 54.7 \% de las y los estudiantes afirmó contar con una beca o bien algún apoyo económico. Salvo en la carrera de derecho, los hombres de la carrera de administración e ingeniería son quienes mayormente cuentan con este tipo de apoyos. En relación con las instituciones a las que pertenecen los estudiantes con becas o apoyos, el EUPriC-CDMX y el EUPUC-GDL son las instituciones que concentran al mayor número de estudiantes (55.8\%).

9 Para caracterizar mejor las diferencias de clase en la investigación se construyó además un Índice de Oportunidad Socioescolar (IOSE) integrado por tres capitales: familiar, escolar y de oportunidad de elegir. Este índice está hecho para diferenciar grupos de estudiantes con distintos volúmenes de capitales (Bourdieu, 1987b) y ubicar a la población en dos posiciones sociales de origen que diferencian a su vez dos estratos: la Posición Social de Origen Baja (PSO), en estratos Muy Desfavorecido (MD) y Desfavorecido (D); y la PSO Alta, que distingue a su vez estratos Favorecido (F) y Muy Favorecido (MF). Para este artículo se decidió trabajar con la pregunta sobre la autoadscripción a la clase porque, como ya se mencionó, se asume que las condiciones de las y los estudiantes de maestría, y sus propios capitales, se han visto modificados a partir de su primera experiencia en la educación superior (en términos de que han experimentado mayor autonomía, que dependen en menor medida de sus padres, etcétera) y que su referencia socioeconómica ya no puede centrarse sólo en el capital familiar. 
La relación entre la autoadscripción de clase y los porcentajes de estudiantes que reciben apoyos económicos o becas indica que son las y los estudiantes del estrato medio bajo quienes concentran el mayor número de apoyos, seguidos por las y los del estrato medio alto. Las principales instituciones en las que se localizan dichos estudiantes son el EUPriC-CDMX, el EUPuCCDMX y el EUPuC-GDL; es decir, los tres Espacios Consolidados del Estudio, dos públicos y un privado.

Sólo $11.49 \%$ de las y los estudiantes dijeron depender económicamente de sus padres. La cantidad de hombres y mujeres en situación de dependencia es igual (48 estudiantes en cada grupo); no obstante, las diferencias por carrera indican que las mujeres que estudian ingeniería dependen en menor medida de sus padres, igual que los hombres de la carrera de derecho. En la carrera de administración, la misma cantidad de hombres y mujeres dijeron depender económicamente de sus familias.

Finalmente, $75.38 \%$ de las y los estudiantes afirmaron estar insertos en alguna actividad laboral. De este porcentaje, $56.45 \%$ fueron hombres, principalmente estudiantes de la carrera de administración. Las principales instituciones a las que pertenecen las y los estudiantes que laboran 40 horas o más son el EUPUC-CDMX, en segundo lugar, el EUPriC-CDMX y en tercero el EUPUC-GDL. Las dos principales clases a las que se autoadscriben la mayoría de las y los estudiantes que trabajan este número de horas, y que juntas concentran $93 \%$ del total, son la clase media baja (55\%) y la media alta (38\%).

\section{Principales resultados}

Para el análisis de los anhelos de las y los estudiantes de maestría de esta muestra se clasificaron las respuestas que dieron a la pregunta de opción múltiple“ ¿Cuáles son sus principales anhelos? en cinco ámbitos: a) social-relacional, b) académico, c) laboral, d) económico y e) experiencial. ${ }^{10}$

10 Para apoyar la clasificación de los anhelos se consideró la propuesta de Arthur Chickering en torno a los diversos ámbitos de desarrollo humano. Aunque esta teoría se utiliza normalmente para analizar la construcción de la identidad de los estudiantes, se considera conveniente para entender los aspectos en los que las y los estudiantes depositan sus anhelos. De acuerdo con Chickering, los estudiantes universitarios transitan por vectores a diferentes ritmos y pueden incluso retornar a algunos que han dejado atrás. Reyes (2010), Soares, Guisande y Almeira (2004) clasifican estos vectores de la siguiente manera: 1) El desarrollo de competencias (intelectuales, sociales e interpersonales) cuyo sentido se manifiesta, de manera concreta, en la confianza con que los estudiantes asumen los retos que se les presentan y en alcanzar objetivos con éxito; 2) La gestión de las emociones, que concierne al desarrollo de la capacidad para reconocer y aceptar las emociones, para poder expresarlas y controlarlas adecuadamente; 3) El desarrollo de la autonomía, que apunta al reconocimiento y aceptación de la importancia de la interdependencia, incluyendo el desarrollo de la independencia emocional e instrumental; 4) El desarroIlo de relaciones interpersonales, vinculadas con la aceptación y la tolerancia de las diferencias individuales, incluyendo el establecimiento de relaciones íntimas; 5) El establecimiento de la identidad, que se refiere a la autoaceptación en un sentido integral: apariencia, género y orientación sexual, continuidad de la identidad en diferentes situaciones e integración; 6) El desarrollo del propósito, importante para la toma de decisiones profesionales, afirmación de intereses personales y el establecimiento de compromisos a largo plazo; 7) El desarrollo de la integridad, "....entendida como la clarificación, compromiso y congruencia de los estudiantes con un conjunto de valores" (Blanco, 2005: 19 en Reyes; 2010). Si bien esta propuesta se ha pensado principalmente para jóvenes de etapas previas al posgrado, se retoma en este trabajo para precisar que los sujetos desarrollan en diferentes ritmos competencias, emociones, relaciones y propósitos que abarcan aspectos concernientes a las relaciones sociales, el ámbito profesional, la autonomía, la identidad, etcétera. Pensando los anhelos como 
Con ello se buscó conocer cómo se configura el ideal de futuro y qué elementos, o bien áreas, poseen mayor peso dentro de los modelos de vida futuros de las y los estudiantes de maestría. A continuación, se detallan las principales respuestas en cada ámbito.

\section{a) Anhelos en el ámbito social-relacional}

Para conocer los anhelos en el ámbito social-relacional, es decir, aquellos anhelos que se insertan en el marco de las relaciones sociales y en la acción en el entorno comunitario, se contemplaron las siguientes respuestas: tener prestigio, conocer gente y hacer relaciones, ayudar en mi comunidad y tener pareja.

En una sociedad en la que, de acuerdo con Veblen (1974), la estima social de una persona se asocia con proezas y hazañas individuales que determinan incluso la propia autoestima, no resulta extraño que el principal anhelo en esta categoría sea, para las y los estudiantes de esta muestra, el tener prestigio. Entre quienes mencionan éste como un aspecto importante de sus vidas, destacan los hombres estudiantes del EUPUC-CDMX y EUPUC-GDL, es decir, los dos principales Espacios Universitarios Públicos considerados en este estudio. La principal carrera en la que se ubican los estudiantes de este grupo es la de administración.

Sin ser un asunto excepcional, conocer gente es el segundo anhelo significativo en esta categoría, toda vez que en México existe la percepción de que ciertas relaciones sociales abren espacio a favores que pueden permitir a una persona obtener empleos, resolver problemas, alcanzar ciertos beneficios, etcétera (Zalpa, Tapia, Martínez, 2014). Este anhelo resulta más importante para los hombres de la carrera de administración en instituciones tales como EUPuCCDMX, EUPUC-GDL y el EUPriC-CDMX; es decir, los tres principales Espacios Universitarios Consolidados del Estudio, con la precisión de que los dos primeros son públicos y el tercero privado.

Llama la atención que sólo 10 de las y los 853 estudiantes de la muestra consideren que es importante ayudar en la comunidad; aunque ello confirma lo que Hirsch y Pérez (2005) han sostenido sobre que las instituciones universitarias necesitan introducir contenidos de formación ética y compromiso social en todas las carreras universitarias. Asimismo, teniendo en cuenta que en las visiones de las universidades de este estudio se considera primordial formar profesionistas con compromiso social, este dato resulta revelador.

Para cinco mujeres y cinco hombres es para quienes resulta importante ayudar a la comunidad. Las instituciones a las que están incorporados son, en primer lugar, el EUPuV- CDMX, el EUPUC-CDMX, el EUPUC-GDL y finalmente el EUPriV-GDL. Seis de estos estudiantes pertenecen a la carrera de derecho y sólo uno a la de administración. Como mencionamos, además, las carreras

parte del proyecto de vida de un sujeto, que se construye articulando la identidad personal-social en una dinámica temporal y de posibilidades, como "un modelo ideal sobre lo que el individuo espera o quiere ser y hacer, que toma forma concreta en la disposición real y sus posibilidades internas y externas de lograrlo, defendiendo su relación hacia el mundo y hacia sí mismo, su razón de ser como individuo en un contexto y tipo de sociedad determinada" (D’Angelo, 2000). 
que cursan los estudiantes son significativas porque alguna de ellas, como derecho en teoría, poseen un estímulo al compromiso social mayor al de otras carreras que tienen un sentido particular en torno a la idea de responsabilidad social.

Tener pareja fue un anhelo mencionado sólo por una mujer de la carrera de derecho de la EUPUV-CDMX, mostrando, como lo sugieren diversos estudios, que la vida en pareja ha dejado de ser un anhelo primordial para los jóvenes en la actualidad. ${ }^{11}$

\begin{tabular}{|c|c|c|c|c|c|c|c|c|c|c|}
\hline \multicolumn{11}{|c|}{ Anhelos en el ámbito social-relacional } \\
\hline \multirow{2}{*}{$\begin{array}{l}\text { Espacio Uni- } \\
\text { versitario }\end{array}$} & \multirow[t]{2}{*}{ Programa } & \multicolumn{2}{|c|}{ 1. Tener prestigio } & \multicolumn{2}{|c|}{$\begin{array}{l}\text { 5. Conocer gente y } \\
\text { hacer relaciones }\end{array}$} & \multicolumn{2}{|c|}{$\begin{array}{l}\text { 11. Ayudar en mi } \\
\text { comunidad }\end{array}$} & \multicolumn{2}{|c|}{ 13. Tener una pareja } & \\
\hline & & Mujer & Hombre & Mujer & Hombre & Mujer & Hombre & Mujer & Hombre & $\begin{array}{l}\text { Total por EU } \\
\text { y programa }\end{array}$ \\
\hline \multirow[t]{4}{*}{ EUPUC-CDMX } & Administración & 13 & 16 & 7 & 12 & o & 1 & 0 & o & 49 \\
\hline & Derecho & 4 & 5 & 1 & 3 & 1 & o & 0 & 0 & 14 \\
\hline & Ingeniería & o & 1 & o & 2 & o & o & 0 & o & 3 \\
\hline & Subtotal & 17 & 22 & 8 & 17 & 1 & 1 & 0 & 0 & 66 \\
\hline \multirow[t]{4}{*}{ EUPUV-CDMX } & Administración & o & o & o & o & o & o & o & 0 & 0 \\
\hline & Derecho & 0 & 1 & o & 1 & 1 & 1 & 1 & 0 & 5 \\
\hline & Ingeniería & o & 4 & o & 1 & o & 1 & 0 & 0 & 6 \\
\hline & Subtotal & 0 & 5 & 0 & 2 & 1 & 2 & 1 & 0 & 11 \\
\hline \multirow[t]{4}{*}{ EUPriC-CDMX } & Administración & 9 & 13 & 5 & 10 & 0 & o & 0 & o & 37 \\
\hline & Derecho & 6 & 2 & o & 1 & 2 & o & o & 0 & 11 \\
\hline & Ingeniería & o & 4 & 0 & 3 & o & o & o & o & 7 \\
\hline & Subtotal & 15 & 19 & 5 & 14 & 2 & 0 & 0 & 0 & 55 \\
\hline \multirow[t]{4}{*}{ EUPriV-CDMX } & Administración & 6 & 3 & 0 & 2 & o & o & o & 0 & 11 \\
\hline & Derecho & 0 & 7 & 0 & 0 & 0 & 0 & 0 & 0 & 7 \\
\hline & Ingeniería & 2 & 6 & 0 & 0 & 0 & 0 & 0 & 0 & 8 \\
\hline & Subtotal & 8 & 16 & 0 & 2 & 0 & 0 & 0 & 0 & 26 \\
\hline \multirow[t]{4}{*}{ EUPUC-GDL } & Administración & 4 & 16 & 3 & 8 & 0 & o & 0 & 0 & 31 \\
\hline & Derecho & 4 & 12 & 2 & 2 & o & o & o & 0 & 20 \\
\hline & Ingeniería & o & 2 & 0 & 4 & o & o & 0 & 0 & 6 \\
\hline & Subtotal & 8 & 30 & 5 & 14 & 0 & 0 & 0 & 0 & 57 \\
\hline \multirow[t]{4}{*}{ EUPriV-GDL } & Administración & 0 & 3 & 0 & 0 & o & o & 0 & 0 & 3 \\
\hline & Derecho & 0 & 1 & 0 & o & 1 & o & o & 0 & 2 \\
\hline & Ingeniería & 0 & 2 & 0 & o & o & 1 & o & 0 & 3 \\
\hline & Subtotal & 0 & 6 & 0 & 0 & 1 & 1 & 0 & 0 & 8 \\
\hline Total & & 48 & 98 & 18 & 49 & 5 & 5 & 1 & 0 & 224 \\
\hline
\end{tabular}

11 En la pregunta sobre el estado civil de las y los encuestados se observa que $68 \%$ dice estar soltera/o, 30\% casado o en unión libre y sólo $1 \%$ divorciada/o, separada/o o viuda/o. 


\section{b) Anhelos en el ámbito académico}

Para conocer los principales anhelos en el ámbito académico se contemplaron las siguientes respuestas: Estudiar un doctorado, Ser profesor/a y Desarrollar investigación. En esta categoría el principal anhelo fue el de estudiar un doctorado. Aunque con una diferencia pequeña, nuevamente son los hombres de la muestra quienes más aluden a su intención de llegar a este grado; la principal carrera a la que pertenecen estos estudiantes es la de derecho. Entre este grupo sobresalen también las y los estudiantes que pertenecen al principal Espacio Universitario Público, es decir el EUPUC-CDMX, y el EUPuV-CDMX. Llama la atención que en el EUPriV-GDL ninguno de los estudiantes participantes en esta muestra consideró relevante este anhelo.

Ser profesor es el segundo anhelo más mencionado en este ámbito. De los seis estudiantes que lo eligieron, cuatro pertenecen a la EUPriV-GDL, dos al EUPUC-CDMX y dos al EUPuV-CDMX. De estos estudiantes cuatro son hombres y sólo dos mujeres. Es importante desarrollar investigación sólo para las y los estudiantes de dos Espacio Universitarios de la Ciudad de México: el EUPUC-CDMX (2) y el EUPuV-CDMX (1). Considerando el total de respuestas a esta pregunta, sin separarlas por ámbitos, podemos observar que sólo $6.68 \%$ de las y los estudiantes manifiestan la intención de estudiar un doctorado, sólo $0.7 \%$ desea ser profesor y $0.4 \%$ desarrollar investigación. Estos datos podrían interpretarse a la luz de que en carreras como administración e ingeniería se valora más la experiencia laboral que la académica y que, como veremos a continuación, para las y los estudiantes de esta muestra resultan mucho más importantes los anhelos laborales que los académicos. 


\begin{tabular}{|c|c|c|c|c|c|c|c|c|}
\hline \multicolumn{9}{|c|}{ Anhelos en el ámbito académico } \\
\hline \multirow[b]{2}{*}{$\begin{array}{l}\text { Espacio Uni- } \\
\text { versitario }\end{array}$} & \multirow[b]{2}{*}{ Carrera } & \multicolumn{2}{|c|}{$\begin{array}{l}\text { 10. Estudiar un } \\
\text { doctorado }\end{array}$} & \multicolumn{2}{|c|}{ 12. Ser profesor/a } & \multicolumn{2}{|c|}{$\begin{array}{l}\text { 16. Desarrollar } \\
\text { investigación }\end{array}$} & \multirow[b]{2}{*}{$\begin{array}{l}\text { Total por EU } \\
\text { y programa }\end{array}$} \\
\hline & & Mujer & Hombre & Mujer & Hombre & Mujer & Hombre & \\
\hline \multirow{4}{*}{$\begin{array}{l}\text { EUPuC- } \\
\text { CDMX }\end{array}$} & Administración & 2 & 4 & 1 & 1 & 2 & 0 & 10 \\
\hline & Derecho & 6 & 6 & 0 & 0 & 0 & 0 & 12 \\
\hline & Ingeniería & 0 & 1 & 0 & 0 & 0 & 1 & 2 \\
\hline & Subtotal & 8 & 11 & 1 & 1 & 2 & 1 & 24 \\
\hline \multirow{4}{*}{$\begin{array}{l}\text { EUPuV- } \\
\text { CDMX }\end{array}$} & Administración & 1 & 2 & 0 & 1 & 1 & 0 & 5 \\
\hline & Derecho & 5 & 5 & 1 & 0 & 0 & 0 & 11 \\
\hline & Ingeniería & 3 & 3 & 0 & 0 & 0 & 0 & 6 \\
\hline & Subtotal & 9 & 10 & 1 & 1 & 1 & 0 & 22 \\
\hline \multirow{4}{*}{$\begin{array}{l}\text { EUPriC- } \\
\text { CDMX }\end{array}$} & Administración & 0 & 0 & 0 & 0 & 0 & 0 & 0 \\
\hline & Derecho & 2 & 1 & 0 & 0 & 0 & 0 & 3 \\
\hline & Ingeniería & 0 & 0 & 0 & 0 & 0 & 0 & 0 \\
\hline & Subtotal & 2 & 1 & 0 & 0 & 0 & 0 & 3 \\
\hline \multirow{4}{*}{$\begin{array}{l}\text { EUPriV- } \\
\text { CDMX }\end{array}$} & Administración & 0 & 0 & 0 & 0 & 0 & 0 & 0 \\
\hline & Derecho & 1 & 1 & 0 & 0 & 0 & 0 & 2 \\
\hline & Ingeniería & 1 & 1 & 0 & 0 & 0 & 0 & 2 \\
\hline & Subtotal & 2 & 2 & 0 & 0 & 0 & 0 & 4 \\
\hline \multirow[t]{4}{*}{ EUPUC-GDL } & Administración & 1 & 2 & 0 & 0 & 0 & 0 & 3 \\
\hline & Derecho & 3 & 2 & 0 & 0 & 0 & 0 & 5 \\
\hline & Ingeniería & 1 & 3 & 0 & 0 & 0 & 0 & 4 \\
\hline & Subtotal & 5 & 7 & 0 & 0 & 0 & 0 & 12 \\
\hline \multirow[t]{4}{*}{ EUPriV-GDL } & Administración & 0 & 0 & 0 & 0 & 0 & 0 & 0 \\
\hline & Derecho & 0 & 0 & 1 & 1 & 0 & 0 & 2 \\
\hline & Ingeniería & 0 & 0 & 1 & 1 & 0 & 0 & 2 \\
\hline & Subtotal & 0 & 0 & 2 & 2 & 0 & 0 & 4 \\
\hline Total & & 26 & 31 & 2 & 4 & 3 & 1 & 67 \\
\hline
\end{tabular}

\section{c) Anhelos en el ámbito laboral}

Para comprender los anhelos en el ámbito laboral, es decir, las posiciones que persiguen las y los estudiantes de la muestra en este ámbito, se eligieron las siguientes respuestas: Trabajar en una empresa, Trabajar en el comercio, Ser dueño/a de una empresa, Trabajar en el sector público y Ser un profesionista independiente.

Lo primero que podemos observar es que el principal anhelo en esta categoría es ser dueño/a de una empresa, donde se concentra $77.6 \%$ de las respuestas en este ámbito. En este anhelo sobresalen las y los estudiantes incorporados a la carrera de administración. El perfil laboral de este programa de estudios está, de entrada, creado para ser acorde con este deseo; 
pero también resulta acorde, como lo han enfatizado Santander, Fernández y Yáñez (2016), con el hecho de que en la sociedad actual el énfasis en el emprendimiento constituye una parte fundamental de las políticas de autoempleo y para la superación de la pobreza que se han impulsado desde diferentes medios. Podemos observar incluso que parte de los proyectos de las IES en la actualidad consiste justamente en promover al emprendimiento como un horizonte laboral para el estudiantado.

Los hombres de la muestra son quienes en su mayoría mencionan anhelar ser dueños de una empresa, algo que tampoco resulta extraño en la medida en que el emprendimiento ha estado históricamente localizado en el universo simbólico de lo masculino (Álvarez, Noguera, Urbano, 2012). En términos de las instituciones a las que pertenecen las y los estudiantes de este grupo, podemos notar que destacan aquellos de los dos principales Espacios Universitarios Públicos Consolidados de la muestra, el EUPuC-CDMX y el EUPUC-GDL.

En segundo lugar, se encuentra el anhelo de trabajar en una empresa. A diferencia del anhelo anterior en este sobresalen las mujeres, así como en la siguiente opción que corresponde a trabajar en el sector público. En términos de las instituciones destacan aquí las estudiantes del Espacio Universitario Consolidado Público y el Privado de la Ciudad de México, es decir el EUPUC-CDMX y el EUPriC-CDMX. La carrera a la que principalmente pertenecen estas mujeres es nuevamente la de administración, cuyo perfil profesional está encaminado justamente a laborar en la gestión de empresas de distintos sectores.

Trabajar en el sector público es una opción para sólo $8.4 \%$ del estudiando de la muestra. Por una muy ligera variación son más las mujeres, de la carrera de administración principalmente, quienes eligen esta opción. Las instituciones a las que pertenecen son el EUPuC-CDMX, el EUPUC-GDL y el EUPuV-CDMX; es decir, los dos Espacios Universitarios Consolidados Públicos de la muestra, el de Guadalajara y la Ciudad de México, y el Espacio Público en Vías de Consolidación de la Ciudad de México.

Únicamente dos mujeres y un hombre de la muestra ( $1.1 \%$ del total de esta categoría), eligen como anhelo el ser un profesionista independiente, es decir, trabajador por cuenta propia. Cabe resaltar que, en términos de los ámbitos de los anhelos elegidos, los laborales son los más importantes para las y los estudiantes considerados en la investigación en la medida en que concentran $30 \%$ de las respuestas totales. 


\begin{tabular}{|c|c|c|c|c|c|c|c|c|c|c|c|c|}
\hline \multicolumn{13}{|c|}{ Anhelos en el ámbito laboral } \\
\hline \multirow[t]{2}{*}{$\begin{array}{l}\text { Espacio Uni- } \\
\text { versitario }\end{array}$} & \multirow[t]{2}{*}{ Carrera } & \multicolumn{2}{|c|}{$\begin{array}{l}\text { 2. Trabajar en } \\
\text { una empresa }\end{array}$} & \multicolumn{2}{|c|}{$\begin{array}{l}\text { 4. Trabajar en } \\
\text { el comercio }\end{array}$} & \multicolumn{2}{|c|}{$\begin{array}{l}\text { 6. Ser dueño/a } \\
\text { de una empresa }\end{array}$} & \multicolumn{2}{|c|}{$\begin{array}{l}\text { 8. Trabajar en el } \\
\text { sector público }\end{array}$} & \multicolumn{2}{|c|}{$\begin{array}{l}\text { 15.Ser un profe- } \\
\text { sional indepen- } \\
\text { diente }\end{array}$} & \multirow[b]{2}{*}{$\begin{array}{l}\text { Total por EU } \\
\text { y programa }\end{array}$} \\
\hline & & M & $\mathrm{H}$ & $M$ & $\mathrm{H}$ & $M$ & $\mathrm{H}$ & M & $\mathrm{H}$ & $M$ & $\mathrm{H}$ & \\
\hline \multirow{4}{*}{$\begin{array}{l}\text { EUPUC- } \\
\text { CDMX }\end{array}$} & Administración & 7 & 2 & 1 & 1 & 37 & 28 & 3 & 1 & 0 & 0 & 80 \\
\hline & Derecho & o & 1 & 0 & 0 & 1 & 7 & 1 & 2 & 1 & 0 & 13 \\
\hline & Ingeniería & 0 & 1 & 0 & 0 & 0 & 4 & 0 & 0 & 0 & 0 & 5 \\
\hline & Subtotal & 7 & 4 & 1 & 1 & 38 & 39 & 4 & 3 & 1 & 0 & 98 \\
\hline \multirow{4}{*}{$\begin{array}{l}\text { EUPuV- } \\
\text { CDMX }\end{array}$} & Administración & 0 & 0 & 0 & 0 & 1 & 1 & 1 & 0 & 0 & 0 & 3 \\
\hline & Derecho & 0 & 0 & 0 & 0 & 0 & 1 & 1 & 1 & 0 & 0 & 3 \\
\hline & Ingeniería & 0 & 1 & 0 & 0 & 1 & 3 & 0 & 2 & 0 & 0 & 7 \\
\hline & Subtotal & 0 & 1 & 0 & 0 & 2 & 5 & 2 & 3 & 0 & 0 & 13 \\
\hline \multirow{4}{*}{$\begin{array}{l}\text { EUPriC- } \\
\text { CDMX }\end{array}$} & Administración & 3 & 0 & 0 & 0 & 9 & 15 & 0 & 0 & 0 & 0 & 27 \\
\hline & Derecho & o & 0 & 0 & 0 & 1 & 0 & 1 & 2 & 0 & 0 & 4 \\
\hline & Ingeniería & 3 & 0 & 0 & 0 & 1 & 3 & o & 0 & 0 & o & 7 \\
\hline & Subtotal & 6 & 0 & 0 & 0 & 11 & 18 & 1 & 2 & 0 & 0 & 38 \\
\hline \multirow{4}{*}{$\begin{array}{l}\text { EUPriV- } \\
\text { CDMX }\end{array}$} & Administración & 2 & 2 & 0 & 0 & 7 & 8 & 0 & o & 0 & 0 & 19 \\
\hline & Derecho & 0 & 0 & 0 & 0 & 3 & 1 & o & o & 0 & 0 & 4 \\
\hline & Ingeniería & 0 & 0 & 0 & 0 & 0 & 5 & 0 & 0 & 0 & 0 & 5 \\
\hline & Subtotal & 2 & 2 & 0 & 0 & 10 & 14 & 0 & 0 & 0 & 0 & 28 \\
\hline \multirow[t]{4}{*}{ EUPUC-GDL } & Administración & 2 & 0 & 0 & 2 & 8 & 31 & 2 & 1 & 0 & o & 46 \\
\hline & Derecho & 0 & 0 & 0 & 1 & 5 & 6 & 2 & 1 & 1 & 1 & 17 \\
\hline & Ingeniería & 1 & 1 & 0 & 0 & 2 & 4 & 0 & o & 0 & 0 & 8 \\
\hline & Subtotal & 3 & 1 & 0 & 3 & 15 & 41 & 4 & 2 & 1 & 1 & 71 \\
\hline \multirow[t]{4}{*}{ EUPriV-GDL } & Administración & 0 & 1 & 0 & 0 & 0 & 1 & 0 & 0 & 0 & 0 & 2 \\
\hline & Derecho & 0 & 0 & 0 & 0 & 0 & 0 & 0 & 0 & 0 & o & 0 \\
\hline & Ingeniería & 1 & 0 & 0 & 0 & 0 & 0 & 0 & 0 & 0 & 0 & 1 \\
\hline & Subtotal & 1 & 1 & 0 & 0 & 0 & 1 & 0 & 0 & 0 & 0 & 3 \\
\hline Total & & 19 & 9 & 1 & 4 & 76 & 118 & 11 & 10 & 2 & 1 & 251 \\
\hline
\end{tabular}

\section{d) Anhelos en el ámbito económico}

Para estar al tanto de los anhelos en el ámbito económico se eligieron las siguientes respuestas: Ganar mucho dinero y Alcanzar estabilidad económica. Estas se separaron del ámbito laboral para conocer la relación que tienen las y los estudiantes con las metas de refuerzo social, es decir, aquellas relacionadas con el propósito de obtener aprobación y evitar rechazo en un contexto como el actual, en el que se valoran aspectos como la riqueza, el poder, el éxito, etcétera, altamente vinculados al aspecto económico.

Como puede observarse, el principal anhelo en esta categoría fue alcanzar estabilidad económica. En relación con las instituciones a las que pertenecen las y los estudiantes de este grupo, 
podemos observar que es en los Espacios Universitarios Consolidados, el EUPuC-CDMX y el EUPUC-GDL, donde mayormente se concentran. En términos de la carrera, puede observarse que es el estudiantado de la carrera de administración, seguido por los de derecho, quienes consideran más significante este anhelo.

Ganar mucho dinero es el segundo anhelo elegido en esta categoría. El estudiantado de la carrera de administración es quien mayormente lo elige, y llama la atención que ninguno de la carrera de ingeniería lo haga. En términos de las instituciones destaca que entre las y los estudiantes del EUPuV-CDMX y el EUPriV-GDL no se elige este anhelo. Aunque las variaciones son minúsculas podemos observar, acorde con estudios como el de Delgado et al. (2010), que los hombres tienen una mayor orientación motivacional hacia esta meta de refuerzo social.

\begin{tabular}{|c|c|c|c|c|c|c|}
\hline \multicolumn{7}{|c|}{ Anhelos en el ámbito económico } \\
\hline \multirow[t]{2}{*}{$\begin{array}{l}\text { Espacio Uni- } \\
\text { versitario }\end{array}$} & \multirow[t]{2}{*}{ Programa } & \multicolumn{2}{|c|}{$\begin{array}{l}\text { 7. Ganar mucho } \\
\text { dinero }\end{array}$} & \multicolumn{2}{|c|}{$\begin{array}{l}\text { 9. Alcanzar estabili- } \\
\text { dad económica }\end{array}$} & \multirow[b]{2}{*}{$\begin{array}{l}\text { Total por EU } \\
\text { y programa }\end{array}$} \\
\hline & & Mujer & Hombre & Mujer & Hombre & \\
\hline \multirow{4}{*}{$\begin{array}{l}\text { EUPuC- } \\
\text { CDMX }\end{array}$} & Administración & 0 & 4 & 9 & 7 & 20 \\
\hline & Derecho & 0 & 0 & 5 & 2 & 7 \\
\hline & Ingeniería & 0 & o & 1 & 2 & 3 \\
\hline & Subtotal & 0 & 4 & 15 & 11 & 30 \\
\hline \multirow{4}{*}{$\begin{array}{l}\text { EUPuV- } \\
\text { CDMX }\end{array}$} & Administración & 0 & o & 1 & 2 & 3 \\
\hline & Derecho & 0 & 0 & 6 & 2 & 8 \\
\hline & Ingeniería & 0 & 0 & 2 & 4 & 6 \\
\hline & Subtotal & 0 & o & 9 & 8 & 17 \\
\hline \multirow{4}{*}{$\begin{array}{l}\text { EUPriC- } \\
\text { CDMX }\end{array}$} & Administración & 1 & 1 & 1 & 2 & 5 \\
\hline & Derecho & 0 & 0 & 5 & 1 & 6 \\
\hline & Ingeniería & 0 & 0 & 0 & 2 & 2 \\
\hline & Subtotal & 1 & 1 & 6 & 5 & 13 \\
\hline \multirow{4}{*}{$\begin{array}{l}\text { EUPriV- } \\
\text { CDMX }\end{array}$} & Administración & 1 & 0 & 3 & o & 4 \\
\hline & Derecho & 0 & 0 & 1 & 1 & 2 \\
\hline & Ingeniería & 0 & 1 & 0 & o & 1 \\
\hline & Subtotal & 1 & 1 & 4 & 1 & 7 \\
\hline \multirow[t]{4}{*}{ EUPUC-GDL } & Administración & 0 & 1 & 1 & 5 & 7 \\
\hline & Derecho & 0 & 1 & 3 & 7 & 11 \\
\hline & Ingeniería & 0 & o & 0 & 5 & 5 \\
\hline & Subtotal & 0 & 2 & 4 & 17 & 23 \\
\hline \multirow[t]{4}{*}{ EUPriV-GDL } & Administración & 0 & 0 & 0 & 1 & 1 \\
\hline & Derecho & 0 & 0 & 1 & o & 1 \\
\hline & Ingeniería & 0 & o & 0 & o & o \\
\hline & Subtotal & 0 & 0 & 1 & 1 & 2 \\
\hline Total & & 2 & 8 & 39 & 43 & 92 \\
\hline
\end{tabular}




\section{e) Anhelos en el ámbito experiencial}

Finalmente, en torno a los anhelos en el ámbito experiencial sólo se eligió una respuesta:Tener oportunidades para viajar. A diferencia de otros anhelos, las mujeres son quienes se destacan en esta categoría porque, recuperando el trabajo de Delgado et al. (2010), el viajar —en ocasiones en el contexto de hacer estancia en el extranjero- suele asociarse fuertemente con las metas de logro y aprendizaje que son significativas para las estudiantes.

En términos de las instituciones, son las de dos Espacios Consolidados las que eligen este anhelo, sólo que un privado y un público de la Ciudad de México: el EUPuC-CDMX y el EUPriCCDMX. En relación con los programas de estudio, nuevamente se destacan las estudiantes de la carrera de administración y en este caso las y los estudiantes de ingeniería que en espacios como el EUPriV-GDL no manifiestan como importante este anhelo.

\begin{tabular}{|c|c|c|c|c|}
\hline \multicolumn{5}{|c|}{ Anhelos en el ámbito experiencial } \\
\hline \multirow{2}{*}{$\begin{array}{l}\text { Espacio Uni- } \\
\text { versitario }\end{array}$} & & \multicolumn{2}{|c|}{$\begin{array}{l}\text { 3. Tener oportuni- } \\
\text { dades para viajar }\end{array}$} & \multirow[b]{2}{*}{$\begin{array}{l}\text { Total por EU } \\
\text { y programa }\end{array}$} \\
\hline & & Mujer & Hombre & \\
\hline \multirow{4}{*}{$\begin{array}{l}\text { EUPuC- } \\
\text { CDMX }\end{array}$} & Administración & 44 & 26 & 70 \\
\hline & Derecho & 5 & 1 & 6 \\
\hline & Ingeniería & 1 & 8 & 9 \\
\hline & Subtotal & 50 & 35 & 85 \\
\hline \multirow{4}{*}{$\begin{array}{l}\text { EUPuV- } \\
\text { CDMX }\end{array}$} & Administración & 1 & 0 & 1 \\
\hline & Derecho & 4 & 0 & 4 \\
\hline & Ingeniería & 2 & 2 & 4 \\
\hline & Subtotal & 7 & 2 & 9 \\
\hline \multirow{4}{*}{$\begin{array}{l}\text { EUPriC- } \\
\text { CDMX }\end{array}$} & Administración & 10 & 11 & 21 \\
\hline & Derecho & 11 & 0 & 11 \\
\hline & Ingeniería & 4 & 1 & 5 \\
\hline & Subtotal & 25 & 12 & 37 \\
\hline \multirow{4}{*}{$\begin{array}{l}\text { EUPriV- } \\
\text { CDMX }\end{array}$} & Administración & 12 & 10 & 22 \\
\hline & Derecho & 4 & 3 & 7 \\
\hline & Ingeniería & 4 & 3 & 7 \\
\hline & Subtotal & 20 & 16 & 36 \\
\hline \multirow{4}{*}{ EUPUC-GDL } & Administración & 10 & 17 & 27 \\
\hline & Derecho & 7 & 5 & 12 \\
\hline & Ingeniería & 1 & 3 & 4 \\
\hline & Subtotal & 18 & 25 & 43 \\
\hline \multirow{4}{*}{ EUPriV-GDL } & Administración & 1 & 1 & 2 \\
\hline & Derecho & 0 & 2 & 2 \\
\hline & Ingeniería & 0 & 0 & 0 \\
\hline & Subtotal & 1 & 3 & 4 \\
\hline Total & & 121 & 93 & 214 \\
\hline
\end{tabular}




\section{A manera de cierre}

Como hemos indicado, la capacidad de aspirar supone la habilidad para explorar y compartir ideas $u$ horizontes de futuro en una sociedad específica, supone orientar las acciones de manera realista y entender el presente en relación con su valor para el futuro (Appadurai, 2004), pero también elementos de desigualdad que van configurando dicha capacidad.

En este análisis se tomaron como referencias tres elementos de desigualdad: el Tipo de Espacio Universitario, la carrera y el género. Sobre el primero podemos observar que los tres principales ámbitos donde se ubican los anhelos de las y los estudiantes de la muestra de las seis instituciones consideradas en la investigación son:

\begin{tabular}{|l|l|}
\hline \multicolumn{1}{|c|}{ Espacio Universitario } & \multicolumn{1}{c|}{ Principales anhelos } \\
\hline EUPUC-CDMX & $\begin{array}{l}\text { 1. Anhelos en el ámbito laboral } \\
\text { 2. Anhelos en el ámbito experiencial } \\
\text { 3. Anhelos en el ámbito social }\end{array}$ \\
\hline EUPUV-CDMX & $\begin{array}{l}\text { 1. Anhelos en el ámbito académico } \\
\text { 2. Anhelos en el ámbito laboral } \\
\text { 3. Anhelos en el ámbito experiencial }\end{array}$ \\
\hline EUPriC-CDMX & $\begin{array}{l}\text { 1. Anhelos en el ámbito social } \\
\text { 2. Anhelos en el ámbito laboral } \\
\text { 3. Anhelos en el ámbito experiencial }\end{array}$ \\
\hline EUPriV-CDMX & $\begin{array}{l}\text { 1. Anhelos en el ámbito experiencial } \\
\text { 2. Anhelos en el ámbito laboral } \\
\text { 3. Anhelos en el ámbito social }\end{array}$ \\
\hline EUPUC-GDL & $\begin{array}{l}\text { 1. Anhelos en el ámbito laboral } \\
\text { 2. Anhelos en el ámbito social } \\
\text { 3. Anhelos en el ámbito experiencial }\end{array}$ \\
\hline EUPriV-GDL & $\begin{array}{l}\text { 1. Anhelos en el ámbito social } \\
\text { 2. Anhelos en el ámbito académico } \\
\text { 3. Anhelos en el ámbito experiencial }\end{array}$ \\
\hline
\end{tabular}

En cada uno de los espacios universitarios los anhelos más importantes se ubican en el ámbito laboral, académico y experiencial. En los Espacios Universitarios Públicos Consolidados, como puede observarse, las y los estudiantes ubican sus principales anhelos en el ámbito laboral. Esto puede resultar significativo para la propia valoración que las y los estudiantes hacen sobre los estudios universitarios en la medida en que, a pesar del debilitamiento de la idea, aún se valoran los estudios universitarios como medios para alcanzar la movilidad social y la inserción en el mercado laboral.

Como también puede observarse que las y los estudiantes de dos de los Espacios Universitarios Privados, el consolidado de la Ciudad de México y el de en Vías de Consolidación de Guadalajara, ubican sus principales anhelos en el ámbito social. Como han mostrado algunas investigaciones sobre los motivos por los que estudiantes de ciertos estratos sociales ingresan a las instituciones privadas, este aspecto no resulta extraño toda vez que en el imaginario existe 
la creencia de que el asistir a las universidades privadas tiene como ventaja la posibilidad de conocer gente y hacer redes que puedan proporcionar apoyo incluso para lograr anhelos laborales.

En relación con la carrera, podemos observar que también existe una distinción interesante con respecto a la prioridad que se le da al tipo de anhelos: para las y los estudiantes de la carrera de administración el aspecto laboral, experiencial y social tienen mayor importancia que los deseos en torno al ámbito económico y académico. En cambio, las y los estudiantes de la carrera de derecho priorizan el aspecto social sobre el experiencial y el laboral, dejando también los anhelos económicos y académicos en el último y penúltimo lugar. Los estudiantes de ingeniería ven en los ámbitos laboral y social sus principales anhelos, y como de menor importancia los anhelos económicos. Como mencionamos anteriormente, ello puede interpretarse bajo la luz de que, por ejemplo, en carreras como la de administración se valora en el mercado laboral mucho más la experiencia que la formación académica.

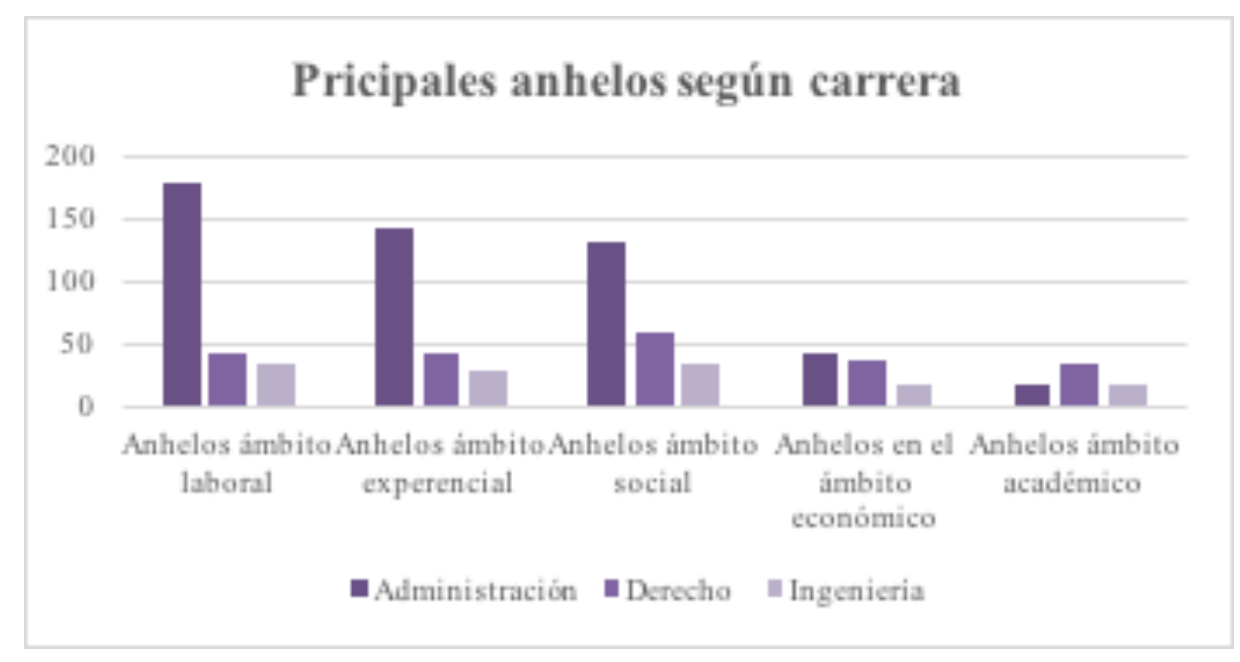

En relación con el sexo de las y los maestrantes podemos observar que para las mujeres el ámbito experiencial se coloca como un anhelo más importante. A diferencia de los hombres, que priorizan el aspecto social, dominado por el deseo de tener prestigio, las mujeres priorizan la posibilidad de tener experiencias tales como viajar. Al igual que los hombres, los anhelos laborales se ubican en un segundo lugar, aunque para ellas los anhelos en el ámbito socialrelacional (a través del prestigio principalmente) ocupan el tercer lugar de preferencia. Igual que en las tendencias por carrera, los anhelos en el ámbito económico y académico ocupan el tercero y cuarto lugar de preferencia entre las y los estudiantes de la muestra. 


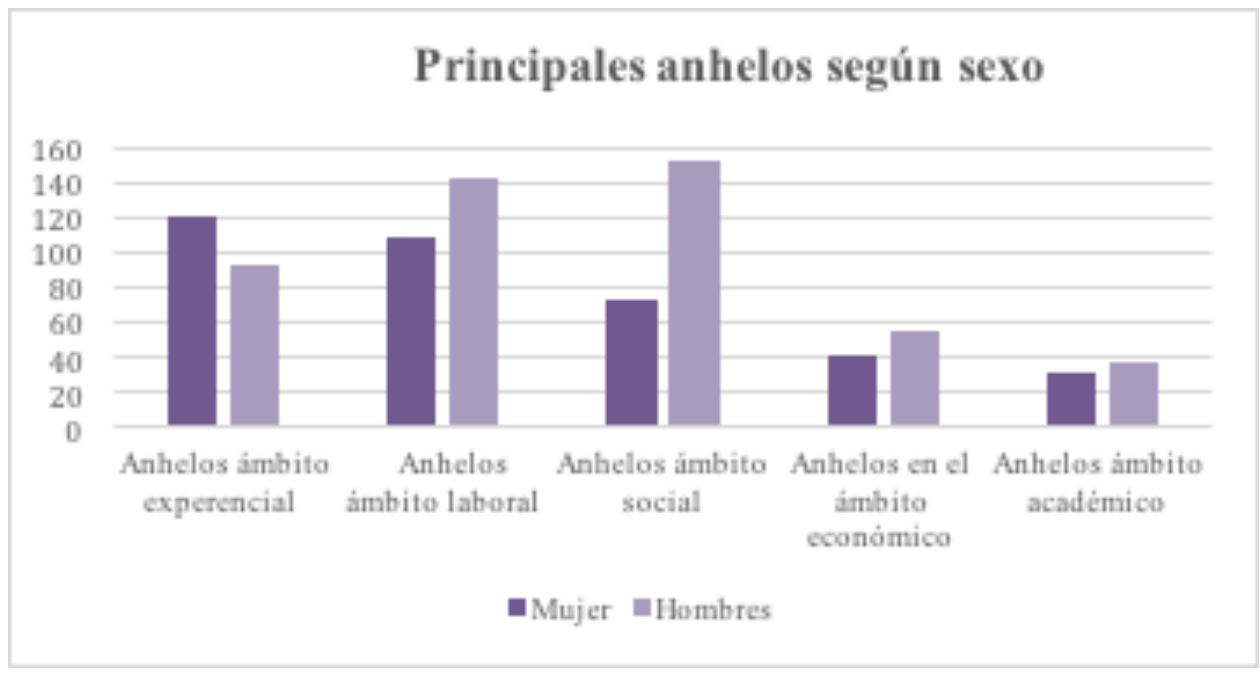

¿Qué sugiere esto? Por un lado, que las y los estudiantes han satisfecho ya la mayoría de sus anhelos académicos y buscan ahora la inserción laboral en un espacio que les permita mantener independencia y autonomía. De acuerdo con los estudios sobre estudiantes de maestría, una importante proporción de aquellos estudiantes que entran a posgrados lo hacen buscando tener una beca y evitar con ello la precariedad laboral; de esta manera, el haber cursado estudios de posgrado significa que tienen - o bien tuvieron-ya un periodo con mayores posibilidades económicas que no desean perder $y$, por lo tanto, el lograr la estabilidad laboral representa para ellas y ellos la posibilidad de mantener seguridad económica y alcanzar el prestigio deseado.

Por otro lado, resulta importante observar que, a través de los anhelos que manifiestan las y los estudiantes de esta muestra, existe una amplia preocupación por el empleo, el reconocimiento y las experiencias. Finalmente, que en relación con el contexto es muy importante pensar los objetivos y visiones de las instituciones de educación superior los anhelos de dichos estudiantes; en la medida en que, como lo sugieren situaciones como la del limitado compromiso social que se observa en las respuestas que analizamos, las IES no sólo son asimétricas y contribuyen a complejizar las desigualdades entre el estudiantado, sino que poco abonan a la formación de personas preocupadas y ocupadas en la atención de los problemas sociales.

\section{Bibliografía}

Álvarez, C.; M. Noguera; D. Urbano (2012). Condicionantes del entorno y emprendimiento femenino. Un estudio cuantitativo en España. Economía Industrial, 1(383), 43-52. https://www. mincotur.gob.es/Publicaciones/Publicacionesperiodicas/Economialndustrial/RevistaEconomialndustrial/383/Claudia\%20Alvarez.pdf 
Ángeles, C. (2016). Brechas de género en la temporalidad individual de la población estudiante de la maestría en México. Tesis. México: El Colegio de la Frontera Norte. https://colef.repositorioinstitucional.mx/jspui/handle/1014/380

Appadurai, A. (2004). The Capacity to Aspire: Culture and the Terms of Recognition. En Rao, V.; M. Walton (eds.). Culture and Public Action. California: Stanford University Press, 58-84.

Barrera, L.; M. Sotelo; J.Vales; D. Ramos (2018). Perspectiva temporal hacia el futuro y rendimiento académico en estudiantes universitarios. Educación y Ciencia 7(49), 37-44. https://www. researchgate.net/publication/344672591 Perspectiva temporal hacia el futuro y rendimiento academico en estudiantes universitarios

Blanco, G. (2005). La experiencia del extracurrículum en estudiantes universitarios. Tesis. México: Universidad de las Américas Puebla. https://www.researchgate.net/publication/37612717 La experiencia del extracurriculum en estudiantes universitarios

Corica, A. (2012). Las expectativas sobre el futuro educativo y laboral de jóvenes de la escuela secundaria: entre lo posible y lo deseable. Última Década, (36), 71-95. https://scielo.conicyt. cl/scielo.php?script=sci arttext\&pid=S0718-22362012000100004

D`Angelo, O. (2000). Proyecto de vida como categoría básica de interpretación de la identidad individual y social. Revista Cubana de Psicología, 17(3), 270-275. http://pepsic.bvsalud.org/ $\mathrm{pdf} / \mathrm{rcp} / \mathrm{v} 17 \mathrm{n} 3 / 08 . \mathrm{pdf}$

Delgado, B.; C. Inglés; J. García; J. Castrejón; A. Valle (2010). Diferencias de género y curso en metas académicas en alumnos de Educación Secundaria Obligatoria. Revista Española de Pedagogía, 68(245), 67-84. https://revistadepedagogia.org//xviii/no-245/diferencias-de-genero-y-curso-en-metas-academicas-en-alumnos-de-educacion-secundaria-obligatoria/101400010122/

Figueroa, A.; L. Padilla; C. Guzmán (2015). La aspiración educativa y la experiencia de ingreso a la educación superior de los estudiantes que egresan de bachillerato en Aguascalientes, México. Pensamiento Educativo. Revista de Investigación Educacional Latinoamericana, 52(1), 18-32. http://www.revistacienciapolitica.cl/index.php/pel/article/view/26075

Führer, A. (2009). Aspiraciones educacionales y laborales de los y las jóvenes que egresan de la educación secundaria en la comuna del Río Hurtado, región de Coquimbo, Chile. Chile: Universidad de Chile. http://www.revistacienciapolitica.cl/index.php/pel/article/view/26075

Gaeta, M.; M. Reyes; M. González; L. García; M. Espinosa; M. Gutiérrez; Y. Benítez (2020). Perspectivas de futuro, patrones de aprendizaje y rendimiento académico en estudiantes universitarios mexicanos. Estudios sobre Educación, (39), 9-31. https://revistas.unav.edu/index.php/ estudios-sobre-educacion/article/view/36670

García-Castro, G.; J. Bartolucci (2007). Aspiraciones educativas y logro académico: un estudio de caso sobre características y condiciones sociales de los estudiantes de la UAM. Revis- 
ta Mexicana de Investigación Educativa, 12(35), 1267-1288. https://www.redalyc.org/ pdf/140/14003507.pdf

Hierro G. (1996) La mujer invisible y el velo de la ignorancia. Metodología para los estudios de género. México: UNAM/Instituto de Investigaciones Económicas.

Hirsch, A. (2005). Actitudes y ética profesional en estudiantes de posgrado en la Universidad de Valencia y en la UNAM. Reencuentro. Análisis de problemas universitarios, (43). https://www. redalyc.org/articulo.oa?id $=34004304$

Kessler, G. (2003). La experiencia escolar fragmentada. Buenos Aires: IIPE-UNESCO.

Luna-Serrano, E.; M. Valle-Espinosa; C. Osuna-Lever (2010). Los rasgos de un "buen profesional", según la opinión de estudiantes universitarios en México. Revista Electrónica de Investigación Educativa, 12, 1-14. http://www.scielo.org.mx/scielo.php?script=sci arttext\&pid=S1607-40412010000300006\&lng=es\&tlng=es

Moyano, F. (2009). La representación social del ser universitario y la práctica académica. XXVII Congreso de la Asociación Latinoamericana de Sociología. VIII Jornadas de Sociología de la Universidad de Buenos Aires. Buenos Aires: Asociación Latinoamericana de Sociología, Universidad de Buenos Aires. https://www.redalyc.org/pdf/3845/384539803008.pdf

Oakley, J.; D. Cocking (2001). Virtue Ethics and Professional roles. Cambridge: Cambridge University Press.

Omar, A.; H. Uribe; M. Aguiar; N. Soares (2005). Perspectivas de futuro y búsqueda de sensaciones en jóvenes estudiantes. Un estudio entre Argentina y Brasil. Revista Latinoamericana de Estudios Educativos, (1-2), 165-180. https://www.redalyc.org/pdf/270/27035206.pdf

PNUD (2000). Desarrollo humano en Chile 2000. Más sociedad para gobernar el futuro. Santiago: PNUD.

Reyes, F. (2010). Los 7 vectores de desarrollo de Chickering y Reisser. Periplos en red. https://periplosenred.blogspot.com/2010/05/los-7-vectores-de-desarrollo-de.html

Santana Vega, L.; L. Feliciano García; A. Santana Lorenzo (2012). Análisis del proyecto de vida en el alumnado de educación secundaria. Revista Española de Orientación y Psicopedagogía, 23(1), 26-38. https://www.redalyc.org/pdf/3382/338230790004.pdf

Santander-Astorga, P.; C. Fernández-Robin; D. Yánez-Martínez (2016). Motivaciones y condicionantes contextuales en el emprendimiento liderado por mujeres chilenas. Revista de Ciencias Sociales 22(2), 63-77. https://dialnet.unirioja.es/servlet/articulo?codigo=5778310

Sellar, S.; T. Gale; S. Parker (2011). Appreciating Aspirations in Australian Higher Education. Cambridge Journal of Education, 41 (1), 37 52. https://www.tandfonline.com/doi/abs/10.1080/03 05764X.2010.549457

Sepúlveda, L.; M.Valdebenito (2014). ¿Las cosas claras? Aspiraciones de futuro y proyecto educativo laboral de jóvenes estudiantes secundarios. Estudios Pedagógicos, XL(1), 243- 261. https:// scielo.conicyt.cl/scielo.php?script=sci abstract\&pid=S0718-07052014000100015\&lng=es \&nrm=iso 
Soares, A.; M. Guisande; L. Almeida (2004). Autonomía y ajuste académico: un estudio con estudiantes portugueses de primer año. International Journal of Clinical and Health Psychology, 7(03). http://redalyc.uaemex.mx/redalyc/pdf/337/33770312.pdf

Tiramonti, G. (2004). La fragmentación educativa y los cambios en los factores de estratificación. En Tiramonti, G. (comp.). La trama de la desigualdad educativa. Buenos Aires: Manantial.

Veblen, T. (1974). Teoría de la clase. México: Fondo de Cultura Económica.

Villa, L.; A. Canales; M. Hamui (2017). Expresiones de las desigualdades sociales en espacios universitarios asimétricos. México: UNAM, ISS, CONACYT.

Zalpa, G.; E. Tapia; J. Reyes (2014). “El que a buen árbol se arrima..." Intercambio de favores y corrupción. Cultura y Representaciones Sociales, 9(17), 149-176. https://www.researchgate.net/ publication/273476488 El que a buen arbol se arrima intercambio de favores y corrupcion 
